\title{
On the theory of ion transfer rates across the interface of two immiscible liquids
}

\author{
R. A. Marcus \\ Noyes Laboratory of Chemical Physics, California Institute of Technology, Pasadena, California 91125
}

(Received 3 March 2000; accepted 27 April 2000)

\begin{abstract}
Ion transfer across the interface of two immiscible liquids involves a mechanism for initiating desolvation from the first liquid, $A$, and concerted solvation by the second, $B$. In the present article a mechanism is considered in which this initiation is facilitated by the ion attaching itself to the tip of a solvent protrusion of $B$ into $A$. (Protrusions have been observed in computer simulations and termed "fingers" or "cones.") It is presumed that the most effective protrusion represents a balance between two opposing effects: the more convex the protrusion the less probable the ion/ protrusion formation but also the less the resistance to extrusion of the intervening liquid between the ion and the surface. An analogy of the latter to hydrodynamics is noted, namely, the more convex the surface the less the frictional force it exerts on the approaching ion. After diffusion in coordinate and solvation space across the interfacial region, the final detachment of the ion from solvent $A$ is assumed to occur from a protrusion of $A$ into $B$. Existing data on ion transfer rates are discussed, including the question of diffusion vs kinetic control. Computer simulations that correspond to the experimental conditions in realistic liquids for measurement of the electrochemical exchange current rate constant $k_{0}$ are suggested. They can be used to test specific theoretical features. With a suitable choice of systems the need (and a major barrier to the simulations) for having a base electrolyte in such simulations can be bypassed. An experiment for the real-time observation of an ion leaving the interface is also suggested. (C) 2000 American Institute of Physics. [S0021-9606(00)50628-X]
\end{abstract}

\section{INTRODUCTION}

The study of ion transfers across the interface of two immiscible liquids has been extensively pursued in recent years (e.g., Refs. 1-18) as has that of electron transfers ${ }^{18-36}$ and of various liquid/liquid interfacial properties. ${ }^{37-48}$ In the present article several factors are considered in formulating a theory of ion transfer across these interfaces. There have been a number of theories of the ion transfer, ${ }^{49-55}$ as well as computer-based insight, ${ }^{56-66}$ and we draw upon some of these studies. There have also been several theoretical studies of electron transfer at these interfaces. ${ }^{67-73}$ Interest in the field of ion transfer itself has stemmed, in part, from its intrinsic interest and, in part, from its role in the areas of liquid-liquid extraction, ${ }^{74}$ ion transport across biological membranes, ${ }^{75}$ and phase transfer catalysis. ${ }^{76}$

Analytic theoretical studies of ion transfers have been various: a stochastic approach, using Eq. (1) below and a potential energy barrier chosen to fit the results, ${ }^{49}$ a related approach without a barrier but with a phenomenological very small diffusion coefficient for crossing the surface, ${ }^{50}$ a lattice gas model to calculate the potential of mean force and thereby an activation barrier, ${ }^{51}$ and a model which includes desolvation/solvation, electrostatic effects and energetics of opening a pore in the interface, in conjunction with an equivalent (the Langevin equation) of Eq. (1) below. ${ }^{55}$ The present approach differs from these analytic approaches in invoking protrusions as a mechanism for initiating the desolvation/solvation and for completing the transfer of the ion across a liquid/liquid interface. It allows for the presence of a barrier, when it occurs.

Experimental results ${ }^{1-18}$ on rates of ion transfer across liquid/liquid interfaces have been obtained by various methods, including AC impedance, potential-step chronofluorometry, ${ }^{8}$ chronoabsorptometry, ${ }^{9}$ and potential modulated reflectance, ${ }^{9}$ the spectroscopic methods being limited to fluorescent or light absorbing ions. The reported experimental results ${ }^{1-18}$ include the absolute values of the rate constants $k_{0}$ for the "exchange" current (current at zero electrochemical Gibbs energy of ion transfer), which are apparently usually about $0.01-0.1 \mathrm{~cm} \mathrm{~s}^{-1}$. These values are of the order of 1000-fold less than those expected from a bulk diffusion constant for crossing the interface. The effect of temperature on the ion transfer rate has been studied, ${ }^{16}$ and increasing evidence of kinetic control at lowered temperatures was seen (kinetic semicircles in the impedance plot).

Other features of the experiments are an apparent independence of $k_{0}$ on the Gibbs energy of transfer of the ion from one liquid to the other, ${ }^{7}$ and the independence or weak dependence of $k_{0}$ on the electrolyte concentration. ${ }^{4,8,11}$ Yet another is the nature of the dependence of the rate constant $k$ for ion transfer on the applied potential across the interface, specifically, that the "Tafel" slope changes rapidly with the applied potential, where studied. ${ }^{4,8}$ Under conditions of small driving force (i.e., $k \cong k_{0}$ ) the slope of the Tafel plot is universally around $0.5 .^{2,3,4,7,8,11}$ However, it has been pointed out $^{6,13}$ that some of the measurements are near the diffusion- 
control limit. We comment later on several results obtained using ac impedance of microinterfaces, which reduce the possibility of diffusion control. There is, of course, the separate issue, which we shall not explore, as to whether what is deduced from the measurements is definitely the charge transfer resistance or some other resistance.

The manuscript is organized as follows: In Sec. II A, relevant time constants are considered regarding kinetic vs diffusion control. In Sec. II B remarks on the hydrodynamics of a body approaching a surface, its analytic theory, and some computer simulations are discussed. The theories are described in Sec. IIC, both for plane interfaces and for interfaces with protrusions. An equation for the latter is Eq. (31). Some approximate numerical estimates are given in Sec. II D, followed by a discussion in Sec. III. Of the two competing mechanisms, flat vs protrusion based, insight into which is dominant can be provided by suitable computer simulations, although a direct real-time observation such as the possibility considered in the final section may be helpful.

\section{THEORY}

\section{A. Relevant time constants}

It is useful to recall the combination of parameters involved in the determination of rate constant $k$ by several of the methods. In the case of ac impedance studies this quantity is [e.g., Eq. (15) of Ref. 4] $k / \sqrt{(2 \omega D)}$, where $\omega$ is the angular frequency of the ac voltage and $D$ is the diffusion constant of the ion in bulk liquid. (Strictly speaking, it should involve $D$ in each of the solvents, by symmetry, but this aspect has only a minor effect on the general conclusions.) If we write $1 / \omega$ as $\tau$, a characteristic time, the parameter is then $k \tau / \sqrt{(2 D \tau)}$, i.e., the ratio of a "reaction distance" traveled in time $\tau$ divided by the root mean square distance traveled in time $\tau$ by the ion diffusing in the liquid. When this ratio becomes substantially larger than unity, the reaction becomes diffusion-controlled. In each case we use below the $k$ inferred from experiment and then see if it satisfies an internal consistency test, namely, see if $k / \sqrt{2 \omega D}$ is not large.

Experimentally, $k$ is estimated from this parameter, e.g., taking a suitable ratio of slope to intercept of a particular plot, or by a fit of a plot of imaginary versus real parts of an impedance. Taking as an example the transfer of a $\mathrm{Cs}^{+}$ across a water $(\mathrm{W}) /$ nitrobenzene $(\mathrm{NB})$ interface, ${ }^{4}$ where the diffusion constant of $\mathrm{Cs}^{+}$in the aqueous phase is 2 $\times 10^{-5} \mathrm{~cm}^{2} \mathrm{~s}^{-1}$, that in the organic phase is $10^{-5} \mathrm{~cm}^{2} \mathrm{~s}^{-1}$, and a typical $\omega$ is between $10 \mathrm{~s}^{-1}$ and $100 \mathrm{~s}^{-1}$, the $k$ estimated from the data is $0.01 \mathrm{~cm} \mathrm{~s}^{-1}$. The parameter $k / \sqrt{(2 \omega D)}$ is then between $1 / 4$ and 1 , suggesting that this $k$ may well be a legitimate $k$, unless the charge transfer impedance has been overwhelmed by impedance due to other sources, there being other factors in the circuitry. For the transfer of tetraethylammonium ion across a W/NB interface, the $k_{0}$ determined from ac impedance studies ${ }^{2,11}$ with macrointerfaces is $0.05-0.15 \mathrm{~cm} \mathrm{~s}^{-1}$ or more ${ }^{5}$ while $D$ is 9.3 $\times 10^{-6} \mathrm{~cm}^{2} \mathrm{~s}^{-1}$ in $\mathrm{W}$ and $4.0 \times 10^{-6} \mathrm{~cm}^{2} \mathrm{~s}^{-1}$ in NB. For an $\omega$ of $10-100 \mathrm{~s}^{-1}$ and using $k=0.15$, the above parameter is about 3-10 (using $D^{\mathrm{W}}$ ). While this latter result does not indicate an overwhelming diffusion-limited rate, it is large enough to warrant caution in the interpretation. We note further that at potentials away from the value where $k=k_{0}$, the average of the forward $\left(k_{f}\right)$ and reverse $\left(k_{r}\right) k$ 's appears instead of $k$ itself, and is larger than $k_{0}$. Results obtained with microinterfaces gave $0.22 \mathrm{~cm} \mathrm{~s}^{-1}$ for this system, ${ }^{6}$ which is a little higher than the above value obtained with macrointerfaces. ${ }^{2,11}$

We consider next a result obtained from potential-step chronofluorometry. ${ }^{8}$ Here, a critical parameter is the ratio $(2 / \sqrt{\pi})\left[k_{f}\left(\sqrt{t / D^{\mathrm{W}}}\right)+k_{r}\left(\sqrt{t / D^{\mathrm{O}}}\right)\right]$, where $\mathrm{O}$ denotes the organic liquid. Again, this quantity is essentially a reaction distance divided by a diffusion distance in time $t$. In the method a ratio of a slope to an intercept is determined. The relevant time range was about $0.01-0.1 \mathrm{~s}$. Using for Eosin $\mathrm{Y}$ dianion $\left(\mathrm{EY}^{2-}\right)$ and a 1, 2-dichloroethane (DCE)/W interface a $D^{\mathrm{W}}=2.1 \times 10^{-6} \mathrm{~cm}^{2} \mathrm{~s}^{-1}, D^{\mathrm{O}}=2.8 \times 10^{-6} \mathrm{~cm} \mathrm{~s}^{-1}$, a $k_{0}=9.5 \times 10^{-3} \mathrm{~cm} \mathrm{~s}^{-1}$, and a $t$ of about $0.1 \mathrm{~s}$ the ratio is about 4.4 , which is not large, though warrants caution.

In a chronoabsorptometric study (total internal reflectance spectroscopy, in conjunction with a potential-step perturbation) the same parameter as that for chronofluorometry appears. ${ }^{9}$ In a study of methyl $\left(\mathrm{MO}^{-}\right)$and ethyl $\left(\mathrm{EO}^{-}\right)$ orange anion transfer across a W/DCE interface the $D_{\mathrm{MO}^{-}}^{\mathrm{W}}$ $=(9 \pm 2) \times 10^{-6} \mathrm{~cm}^{2} \mathrm{~s}^{-1}$, with a $\sqrt{t}$ of about $0.1-0.05 \mathrm{~s}^{1 / 2}$ for $\mathrm{MO}^{-}$and a $k_{0}=(2.5 \pm 0.7) \times 10^{-2} \mathrm{~cm} \mathrm{~s}^{-1}$ the above parameter is about $1-2$, and so, with the caveat mentioned earlier, the $k$ may be legitimate. Ethyl orange $\left(\mathrm{EO}^{-}\right)$displays a similar behavior.

In an analysis of the frequency dependence of potential modulated reflectance, ${ }^{9}$ the critical parameter is given as $4 k_{f} / \sqrt{2 D^{\mathrm{W}} \omega}$. With a $k_{0}$ of $4.5 \times 10^{-2} \mathrm{~cm} \mathrm{~s}^{-1}$ in W/NB, the $D_{\mathrm{MO}}^{\mathrm{W}}=9 \times 10^{-6} \mathrm{~cm}^{2} \mathrm{~s}^{-1}$ for methyl orange, $\omega^{-1 / 2}$ was $0.1-$ $0.05 \mathrm{~s}^{1 / 2}$, and the above quantity is about $2-4$. So once again the $k_{0}$ may be the real $k_{0}$, unmasked by diffusion.

At present, therefore, there are some uncertainties in the interpretation of the data, and I gather that studies at lower competing impedances and at shorter times would be desirable, since one important ratio of the parameters to distinguish diffusion control from kinetic control is $k t / \sqrt{2 D t}$. Other things being equal, it has been noted that studies with microelectrodes can yield valid results for higher $k$ 's, since the solution resistance is relatively less and the replenishment of the transferring ion by diffusion is greater for this geometry (e.g., Ref. 77). With macroelectrodes, the solution resistance can be several hundred times greater than the charge transfer resistance and needs to be carefully compensated.

On the other hand, while with the microelectrodes the charge transfer resistance is considerably increased and can become comparable with the solution resistance, new problems can also arise $;{ }^{77}$ parasitic impedances ${ }^{6}$ and currents at the edges and corresponding disturbance of the interface. ${ }^{78}$ Conclusions presently inferred from studies using ${ }^{6,13}$ microsized liquid/liquid interfaces differ from each other and remain to be resolved. 


\section{B. Remarks on hydrodynamics, analytic theory, and computer simulations}

When a sphere approaches a flat solid surface, the viscous flow resistance increases above that expected from the usual Stokes' formula, because of the difficulties of extrusion of the liquid between the sphere and the surface. ${ }^{79-83}$ Asymptotically the hydrodynamic (macroscopic) resistance varies as $1 / d$, where $d$ is the distance from the leading edge of the sphere to the solid surface. ${ }^{84}$ For an interface which is a free surface, i.e., one which has slip boundary conditions instead of "stick," the resistance is less, but still singular with the distance $d{ }^{84(\mathrm{~b})}$ For a sphere approaching a liquidliquid interface an intermediate behavior is expected since the interface is closer to "slip," but generates a flux in the second liquid and hence an additional dissipation in that fluid.

Thus, from a purely macroscopic viewpoint, apart from any deformation of shape of the interface due to the ion, the ion encounters additional resistance as it approaches a surface closely. (This effect can be shown to be relatively minor for electron transfers. ${ }^{85}$ ) At the molecular level a barrier arises because of the need of replacing one set of solvent molecules by the other in the neighborhood of the ion. The attachment of the ion to the tip of a protrusion, rather than a flat interface, provides a mechanism for facilitating the initial step in a concerted stepwise desolvation of the ion from $A$ and solvation by $B$. In this way, the requirement of displacing several B molecules simultaneously at a flat interface is avoided. Before proceeding to a model, we first recall several results obtained from computer simulations and then suggest where future simulations of appropriate systems closer to actual experimental conditions would be of interest.

Computer simulations of realistic water/organic solvent interfaces ${ }^{58-65}$ yield interfaces which appear as locally sharp ( $\sim 1$ molecular diameter thick). Because of fluctuations (capillary waves) they appear wider $(\sim 10 \AA)$ when examined locally but on a somewhat coarser distance scale. In computer simulations for water/organic interfaces protrusions have been observed by Benjamin and others (and termed "fingers" or "cones") to which a solute ion can become attached. ${ }^{58-61}$ The fingers have extended as much as $\sim 9 \AA$ in the case of water protruding into an organic solvent. Analytic treatments of some aspects of the (less extreme) protrusions have been or can be given in terms of the interfacial tension $\gamma$ and a bulk correlation length $\xi^{58,86,87}$

The computations for realistic liquids have normally been made under conditions where the standard Gibbs energy of ion transfer is large, and without a supporting electrolyte and compensating field to balance this Gibbs ion transfer energy. This balance is needed in order to simulate conditions for the measurement of an experimentally important quantity, the electrochemical exchange current and its associated rate constant $k_{0}$. However, it appears not to be feasible ${ }^{64}$ at present to simulate systems where there is the above balance. Accordingly, as an alternative, it would be useful to simulate systems where the standard Gibbs energy of ion transfer is close to zero, as in $\mathrm{Me}_{4} \mathrm{P}^{+}, \mathrm{PF}_{6}^{-}$or $\mathrm{EtMe}_{3} \mathrm{~N}^{+}$for a water/nitrobenzene system, ${ }^{7}$ or $\mathrm{Et}_{4} \mathrm{~N}^{+}$for a water/1,2-dichloroethane one. ${ }^{88}$ In this case, an applied field and the supporting electrolyte, with its extra complications are no longer needed in a simulation to obtain $k_{0}$. Such a simulation can provide detailed insight into the various mechanistic questions raised below. Just as key insight into electron transfer reactions in solution was obtained from experiments on self-exchange reactions, free from any $\Delta G^{\circ}$ driving force, ${ }^{89}$ analogous benefits can be expected from studies of ion transfers when the counterpart of $\Delta G^{\circ}$ vanishes, i.e., studies of $k_{0}$. To avoid unduly improbable trajectories when this $\Delta G^{\circ} \cong 0$, it would be helpful to use trajectories where the ion is initially thermalized at the center of the interfacial region, and where the number of "recrossings" (or equivalent) is estimated so as to calculate ion transfer rates, as is commonly done now for homogeneous chemical reactions.

In a recent interesting investigation a computer simulation ${ }^{64}$ was made of $\mathrm{Me}_{4} \mathrm{~N}^{+}$crossing a water/ nitrobenzene interface. An applied field of $0.5 \mathrm{~V}$ per $10 \AA$ was used to reduce the trajectory time. We discuss the results in Sec. III. A study of a model (Lennard-Jones) system having zero Gibbs energy of transfer, ${ }^{66(a)}$ yielded interesting results which bear further close analysis, as noted later. It will be interesting to see if the behavior of the protrusions in current computer simulations where the nonzero Gibbs energy of ion transfer has not been balanced by an electric field and electrolyte differs from that in simulations where there is a balance or where the Gibbs energy of transfer is about zero.

The implications of another set of computer simulations but for the approach of an iodide ion to a metal surface is relevant. ${ }^{53}$ In this case the ion displaces the water molecules adsorbed on the surface, and once again there is an extrusion of the solvent molecules between the leading edge of the ion and the metal. A twofold effect occurs, one being the existence of a barrier to reach the transition state for adsorption of the ion on the metal, due to the displacement of the intervening water molecules, and the other being an enhanced friction coefficient $\zeta$, enhanced approximately by a factor of 10 at the separation distance in the transition state. ${ }^{53} \mathrm{Al}-$ though the connection to the behavior on a macroscopic hydrodynamic level for $\zeta$ was not drawn, the computer results do provide clear similarities to the discussion given earlier.

In the calculation in Ref. 53 the frequency dependence $\zeta(\omega)$ was determined in the transition state, and only $\zeta(\omega)$ appropriate to the time spent coming in the TS region is relevant, and it is much smaller than the $\zeta(0)$ at that region. The net result, using Grote-Hynes extension of Kramers' theory of reactions, for the adsorption rate is the near cancellation of two effects, yielding a result which differs only about 30\%-40\% for TS theory. Thus, for the calculations of a rate constant $k_{\text {assn }}^{A}$ below we shall simply use TST theory but note that there may be a barrier in the TST, $w_{A}^{P \dagger}$ or $w_{A}^{\dagger}$ in Eqs. (20) and (25), for the displacement of some solvent molecules.

\section{Theory}

We first recall a phenomenological treatment for ion transfer across the liquid-liquid interface ${ }^{50}$ In that approach a small but finite diffusion constant was assumed for cross- 
ing the interface, taken as flat. The actual mechanism was not specified. The approach in Ref. 50 can be regarded as a special case of the following analysis [cf. Eq. (7) below].

We denote by $c_{i}(z, t)$ the concentration of attached ions at a distance $z$ normal to the local mean interfacial plane (number per unit area per unit $z$ ). The $c_{i}(z, t)$ is assumed to satisfy in the interfacial region the usual diffusion/reaction equation (cf. also Refs. 49 and 56),

$$
\frac{\partial c_{i}}{\partial t}=\frac{\partial}{\partial z}\left(D \frac{\partial c_{i}}{\partial z}\right)+\frac{\partial}{\partial z}\left(\frac{D c_{i}}{k_{B} T} \frac{d \bar{\mu}}{d z}\right)=-\frac{\partial J}{\partial z},
$$

where $D(z)$ is the diffusion coefficient, $\bar{\mu}$ is the electrochemical potential of the ion,

$$
\bar{\mu}(z)=\mu(z)+e \phi(z),
$$

$e$ is the charge of the ion, $\varphi(z)$ is the electrostatic potential at $z$, and $J$ is the flux of $c_{i}$,

$$
J=-D \frac{\partial c_{i}}{\partial z}-\frac{D c_{i}}{k_{B} T} \frac{d \bar{\mu}}{d z} .
$$

A rate constant $k_{\text {rate }}$ can be defined by

$$
J=k_{\text {rate }} c\left(z_{i}\right)=k_{\text {app }} c_{A},
$$

where $k_{\text {app }}$ is the apparent rate constant calculated using the bulk concentration $c_{A}$ of the ion in the initial solvent $A$, and $c\left(z_{i}\right)$ denotes the concentration of the unattached ion at any point $z_{i}$ just outside the interfacial region. The $c_{A}$ and $c\left(z_{i}\right)$ can differ because of electrostatic or other effects (work required to bring ion from bulk $A$ to $z_{i}$ ). The flux $J$ is given by Eq. (3) and equally by Eq. (4). In fact, this equality serves as a boundary condition at $z=z_{i}$ for the integration of Eq. (1). If the flux $J$ were time-dependent, then $c\left(z_{i}\right)$ could be $c\left(z_{i}, t\right)$ and $k_{\text {app }}$ could be $k_{\text {app }}(t)$.

In a steady-state approximation, $\partial c_{i} / \partial t=0$, and Eq. (1) then yields a constant value for the flux $J$. Integration of Eq. (3) from $z_{i}$, the point of entrance to the interfacial region, to $z_{f}$, the point of departure from it, yields the usual result

$$
k_{\text {rate }}=k_{\text {diff }}=1 / \int_{z=z_{i}}^{z_{f}}\left[e^{\left[\bar{\mu}(z)-\bar{\mu}\left(z_{i}\right)\right] / k_{B} T} / D\right] d z .
$$

We recall that a linear profile model has been used by Kakiuchi for $\bar{\mu}(z)$, with $D(z)$ being treated as a constant. ${ }^{50}$ In the present notation the linear profile for $\bar{\mu}(z)$ is

$$
\bar{\mu}(z)-\bar{\mu}\left(z_{i}\right)=\left[\bar{\mu}\left(z_{f}\right)-\bar{\mu}\left(z_{i}\right)\right]\left(z-z_{i}\right) /\left(z_{f}-z_{i}\right)
$$

and the integral in Eq. (5) yields

$$
k_{\mathrm{diff}}=\frac{u e^{-u}}{\sinh u} \frac{D}{z_{f}-z_{i}},
$$

where

$$
u=\left[\bar{\mu}\left(z_{f}\right)-\bar{\mu}\left(z_{i}\right)\right] / 2 k_{B} T .
$$

The value of $k_{\text {rate }}$ at zero driving force $(u=0), k_{\text {rate }}^{0}$, is

$$
k_{\text {rate }}^{0}=D /\left(z_{f}-z_{i}\right) \quad(u=0) .
$$

For an effective thickness of the interface of the order of $10 \AA$ and a rate constant $k_{0}$ of the order of $0.1 \mathrm{~cm} \mathrm{~s}^{-1}$, a phenomenological $D \sim 10^{-8} \mathrm{~cm}^{2} \mathrm{~s}^{-1}$ is required instead of the usual value of bulk solution of the order of $10^{-5} \mathrm{~cm}^{2} \mathrm{~s}^{-1}$.
One question is how can the desolvation of the ion from solvent $A$ and solvation by the second solvent $B$ be initiated. When an ion moves through a bulk liquid there is a tendency for neighbors to persist, as reflected in the well-known "cage effect" of reactants in solution. ${ }^{90}$ This cage effect is considerable in bulk solution. It is reflected in the ratio of $Z_{\mathrm{eq}}$ the equilibrium encounter rate constant, ${ }^{91} \cong g 10^{11} \mathrm{M}^{-1} \mathrm{~s}^{-1}$ [upon including $g$, a hard sphere radial distribution function at contact, with a value of about 5 (Ref. 9)] and the diffusion rate constant, $k_{D} \cong 4 \pi D R, \sim 10^{10} \mathrm{M}^{-1} \mathrm{~s}^{-1}$, where $R$ is the distance between the centers of two neighbors at contact. The ratio $Z_{\text {eq }} / k_{D}$ could be interpreted as a number of collisions with the cage wall before escape, $\sim 10 g$, i.e., $\sim 50$. The separation difficulty is even greater when an escaping neighbor is sandwiched between its neighbor and a flat surface. When an ion enters the second (immiscible) liquid it is forced to change its neighbors because of the repulsions of the unlike ("immiscible') solvent molecules. Thus, a very small transition probability, perhaps of the order of 1/50 or, worse, or if two $A$ molecules need to be displaced by $B$, a probability of $\sim 1 / 2500$, is needed to initiate a penetration of a flat interface, in the absence of deformation.

One mechanism for initiating and completing the desolvation/solvation is the following, motivated by the work on protrusions. We recognize, nevertheless, that computational studies under conditions of zero-electrochemical free energy ion transfer would be useful in ascertaining which of the following factors are dominant in controlling the rate of ion transfer across the interface:

(1) To cross the interface, an ion initially in solvent $A$ first attaches itself to a protrusion of solvent $B$, as depicted schematically in Fig. 1. The probability density (per unit $h$ ) of finding a protrusion of height $h$ is denoted by $P(h)$. This probability density may be influenced by the proximity of the attaching ion, and in that case can be written as $P\left(h, z_{i}\right)$. Alternatively, as discussed later, it can be incorporated into attachment and detachment rate constants which multiply $P(h)$. The $h$ is treated as a signed quantity, negative when the protrusion extends into $A$ and positive when it extends into $B$. It was noted earlier that the attachment of the ion to the tip is a convenient way of initiating the solvation by $B$ and desolvation from A.

(2) The attachment of the ion to the tip of the protrusion of solvent $B$ has a "bimolecular' attachment rate $k^{A} c\left(z_{i}\right) P\left(h_{i}\right)$, where the value of $h$ at the point of attachment is denoted by $h_{i}$, and $c\left(z_{i}\right)$ is the concentration of the unattached ion at $z_{i}$, where $z_{i}=h_{i}-a$, $a$ being the ionic radius. The most probable value of $h_{i}$ depends on factors such as the probability of initiation of solvation by $B$ as a function of $h_{i}$ and the probability density $P\left(h_{i}\right)$ : when $h_{i}$ is about zero, the local interface is flat and too many $A$ molecules may have to be displaced simultaneously by $B$ molecules in the initiation process, even though $P\left(h_{i}\right)$ itself is large when $h_{i} \cong 0$. There is also a rate constant $k_{-}^{A}$ for detachment of the ion back into bulk $A$.

(3) Once attached the ion diffuses across the interface to- 
Ion Transfer Across Immiscible Liquid/Liquid Interface
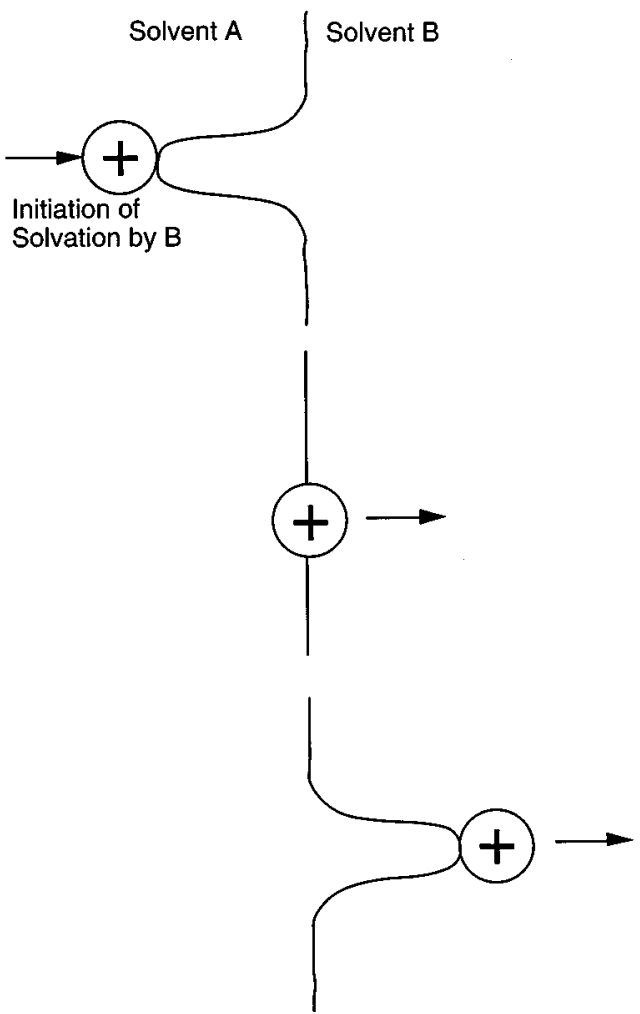

FIG. 1. Schematic plot of an ion in solvent $A$ approaching a protrusion from solvent $B$ at the $A / B$ interface, passing through the interface and exiting into solvent $B$ from another protrusion, now of $B$ into $A$.

ward solvent $B$. The recession of the protrusion presumably assists the solvation of the ion by the new solvent $B$, and this solvation and the desolvation from $A$ continues as the ion diffuses across the interface. The ionic motion during this phase is described approximately by a differential equation for the diffusive motion, influenced by the changing solvation as well as by the changing electrostatic potential $\phi(z)$. It is a diffusion in solvation/ position space, as in Fig. 2

(4) The chemical potential of the ion $\mu(z)$ is taken to depend on the instantaneous value of $z$, counted relative to

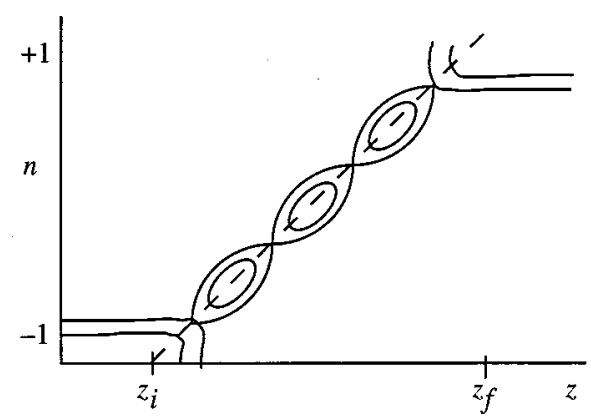

FIG. 2. Schematic plot of free energy contours in solvation-spatial coordinate space for an ion crossing the interfacial region. The ion becomes attached at a protrusion of solvent $B$ into solvent $A$ at $z_{i}$ and detached at $z_{f}$ from a protrusion of $A$ into $B$. The coordinate $n$ is a solvation coordinate, such as that noted in the text, and describes a concerted desolvation of the ion from $A$ and solvation by $B$. the value $z_{i}$ where it became attached. As $z$ increases from $z_{i}$, the solvation of the ion and hence $\mu(z)$ changes, as indicated

(5) At some point, when the change of solvation is nearly complete, the ion begins to detach itself from the interface, which may now be from the tip of a protrusion of solvent $A$ extending into solvent $B$. The ion detaches with a rate $k_{-}^{B} c_{i}\left(z_{f}\right) P\left(h_{f}\right)$, where $z_{f}$ is the value of $z$ at the detachment point $\left(z_{f}=h_{f}+a\right) ; P\left(h_{f}\right)$ is the probability density (i.e., per unit $h_{f}$ ) of this protrusion, which may provide the easiest route for the desolvation of the last $A$ molecules from the ion. This $P\left(h_{f}\right)$ can be written as $P\left(h_{f}, z_{f}\right)$ or, instead, the effect of the nearby ion can be incorporated into $k_{-}^{B}$ which multiplies it in Eq. (13) below. In any system where some $A$ molecules are retained for some time after entering bulk $B$, an appropriate modification of the scheme can be used.

(6) If there is any free energy barrier to actually crossing the interface, it can be incorporated in the $\bar{\mu}(z)$ in Eq. (1), but we focus here on the other factors.

The overall process can be represented schematically as

$$
A_{n_{A}} I+n_{B} B \rightarrow n_{A} A+I B_{n_{B}},
$$

where $I$ denotes the ion and where the $n_{A}$ and $n_{B}$ denote some measure of the solvation, e.g., numbers of nearest solvent molecule neighbors of the ion.

The probability density of a fluctuation of height $h$ of a protrusion of one solvent extending into the other, $P(h)$, in the absence of an approaching ion can be written as

$$
P(h)=e^{-F(h) / k_{B} T} / \int_{-\infty}^{\infty} e^{-F(h) / k_{B} T} d h,
$$

where $F(h)$ is the free energy of formation of the fluctuation (protrusion) and, as noted earlier, $h<0$ describes $B$ protruding into $A$, and $h>0$ for $A$ protruding into $B$. For small fluctuations, $F(h)$ is a quadratic function of $h$. The local mean square value $\left\langle h^{2}\right\rangle$ has been obtained from computer simulations $^{58}$ and has also been estimated from the interfacial (surface) tension using capillary wave theory. The relaxation behavior of these fluctuations is also available from the computer simulations and from expressions from capillary wave theory for the damping behavior. The number of such protrusions per unit area $\sigma$ is, for the moment, assumed to be of the order of $1 / \xi^{2}, \xi$ being the bulk correlation length $(4 \AA$ in Ref. 58). ${ }^{92}$

We again use Eq. (1), where, in the present model, we let $t=0$ be the time of attachment. Then the boundary conditions on Eq. (1) for the flux $J$ of the ion motion are

$$
\begin{aligned}
& J\left(z_{i}, 0\right)=\left[k^{A} c\left(z_{i}\right)-k_{-}^{A} c_{i}\left(z_{i}\right)\right] P\left(h_{i}\right) \delta h_{i} \quad\left(z_{i}<0\right), \\
& J\left(z_{f}, t\right)=k_{-}^{B} c_{i}\left(z_{f}\right) P\left(h_{f}\right) \delta h_{f} \quad\left(z_{f}>0\right),
\end{aligned}
$$

where the attachment and detachment occur in the intervals $\left(h_{i}, h_{i}+\delta h_{i}\right)$ and $\left(h_{f}, h_{f}+\delta h_{f}\right)$, respectively.

This flux is positive since it proceeds from $z<0$ to $z$ $>0$. As noted earlier, the position of the ion at the point of attachment $z_{i}$ is related to $h_{i}\left(z_{i}=h_{i}-a\right)$, and $z_{f}$ is the value of $z$ at the point of detachment, $z_{f}=h_{f}+a$. Ultimately, one 
would obtain the solution, replace $\delta h_{i}$ and $\delta h_{f}$ in it by $d h_{i}$ and $d h_{f}$, and integrate over all possible points of attachment $h_{i}$ and of detachment $h_{f}$. Since $P(h)$ occurs in Eqs. (12) and (13) as a product with a rate constant, the effect of the nearby ion on $P\left(h_{i}\right)$ or $P\left(h_{f}\right)$ can be included, as indicated earlier, equivalently in the attachment and detachment rate constants there.

The units of $k^{A}$ in Eq. (12), the "bimolecular" rate constant for the attachment of the ion in $A$ to a protrusion of $B$ extending into bulk $A$, are $\mathrm{cm}^{3} \mathrm{~s}^{-1}$. The $k_{-}^{A}$ in Eqs. (12) is the rate constant for the detachment of the ion from the protrusion into bulk $A$ and has units of $\mathrm{cm} \mathrm{s}^{-1}$, as does the $k_{-}^{B}$ in Eq. (13).

The $D(z)$ in Eq. (1), written as a diffusion constant in ordinary space, now actually represents a diffusion constant in a combined space: spatial and solvational. During the diffusion in Eq. (1) the solvation changes from being dominantly $A$ to dominantly $B$. If we denote some solvation coordinate by $n$, for example a difference in solvation numbers of $A$ and $B$, e.g., in the number of nearest solvent molecule neighbors of the ion $n_{A}$ and $n_{B}$, divided by their sum, $\left(n_{B}\right.$ $\left.-n_{A}\right) /\left(n_{A}+n_{B}\right)$, this $n$ will vary from -1 to +1 when solvation by $A$ changes to solvation by $B$.

When there are activation barriers to this stepwise change of solvation, the contours of a free energy surface in $(n, z)$ space might resemble those sketched in Fig. 2, where successive barriers to the change of solvation are indicated by saddle-points in the figure. The free energy contours in the figure include a contribution due to the initial and final protrusions. When there is a local solvation equilibrium at each position $z$ of the ion in the interfacial region, its path in $(n, z)$ space is that of the dashed line there. If, because of a large electrochemical free energy driving force, the ion goes rapidly into $B$, e.g., along a path in Fig. 2 which emerges at some $n<1$, the desolvation from $A$ has not had sufficient time to be completed. Such an effect has been seen in a simulation. ${ }^{64}$ It can be included by conversion of Eq. (1) to a partial differential/difference equation in position/solvation space.

While a detailed calculation based on Eqs. (1) and (12)(13) would ultimately involve an integration over $h_{i}$ and over $h_{f}$, since all values contribute, we shall suppose as one approximation that the attachment of the finger of $B$ to the ion occurs largely at some $h_{i}$, denoted now simply by $h_{i}$, with width of contributions, $\Delta h_{i}$, and that similarly the detachment occurs largely at some particular $h_{f}$, denoted now by $h_{f}$, with a width $\Delta h_{f}$. This $h_{i}$ occurs at the maximum of the right-hand side of Eq. (12) and the $h_{f}$ occurs at that for Eq. (13). Equation (1) is then solved using these equations as boundary conditions. For simplicity, a steady-state solution, $\partial c_{i} / \partial t=0$, will again be used for $z$ 's in or near the $\left(z_{i}, z_{f}\right)$ interval in the present article and so the flux $J$ in Eq. (3) is now a constant. Using the boundary conditions (12) and (13) at $z=z_{i}$ and $z_{f}$ we have

$$
\begin{aligned}
& J=\left[k^{A} c_{i}\left(z_{i}\right)-k_{-}^{A} c_{i}\left(z_{i}\right)\right] P\left(h_{i}\right) \Delta h_{i}, \\
& J=k_{-}^{B} c_{i}\left(z_{f}\right) P\left(h_{f}\right) \Delta h_{f} .
\end{aligned}
$$

Integration of Eq. (3) between $z_{i}$ and $z_{f}$ and introduction
TABLE I. List of free energy terms used.

\begin{tabular}{ll}
\hline \hline $\bar{\mu}_{A}$ & $\bar{\mu}$ of the ion in bulk $(z=-\infty)$ \\
$\bar{\mu}\left(z_{i}\right)$ & $\bar{\mu}$ of the unattached ion just outside $z_{i}$ \\
$w_{A}^{\dagger}$ & activation free energy, if any, on going from unattached in \\
& $A$ to attached ion,apart from other free energy \\
& terms in Eq. (25) \\
$\bar{\mu}\left(z_{f}\right)$ & $\bar{\mu}$ of the ion near $z_{f}$ just after detachment \\
$w_{B}^{\dagger}$ & activation free energy, if any, for ion on going from \\
& unattached in $B$ to attached ion, apart from other free \\
$\bar{\mu}_{B}$ & energy terms in Eq. (30) \\
$w_{A}^{P \dagger}, w_{B}^{P \dagger}$ & $\bar{\mu}$ of the ion in bulk $B(z=+\infty)$ \\
\hline \hline
\end{tabular}

of Eqs. (14)-(15) for the value of $J$, yields equations for $c_{i}\left(z_{i}\right)$ and $c_{i}\left(z_{f}\right)$. The rate constant $k_{\text {rate }}$ in the rate expression for ion transfer, $k_{\text {rate }} c\left(z_{i}\right)$, letting $z_{i}$ now denote the most probable $z_{i}$ in Eq. (4), is found to be

$$
\frac{1}{k_{\text {rate }}}=\frac{1}{k_{\mathrm{assn}}^{A}}+\frac{1}{K_{\mathrm{eq}}^{A} k_{\mathrm{diff}}}+\frac{1}{K_{\mathrm{eq}}^{B} k_{\mathrm{diss}}^{B}} .
$$

This $k_{\text {rate }}$ like the $k_{\text {rate }}$ for the phenomenological model in Eq. (5), appears as a $k_{f}$ (in one notation) in expressions for equivalent circuits for the various types of experimental measurements. The diffuse double layer which lies outside $z_{i}$ and outside $z_{f}$ contributes to those equivalent circuits in the form of an impedance. In Eq. (16), $k_{\text {assn }}^{A}$ is the attachment or association rate constant,

$$
k_{\text {assn }}^{A}=k^{A} P\left(h_{i}\right) \Delta h_{i} .
$$

Because of the definition of $k_{\text {rate }}$ in Eq. (4), neither $k^{A}$ nor $K_{\text {eq }}^{A}$ or $K_{\text {eq }}^{B}$ contains the factor $\exp \left\{-\left[\bar{\mu}\left(z_{i}\right)-\bar{\mu}_{A}\right] / k_{B} T\right\}$ (Table I) relating $c\left(z_{i}\right)$ to $c_{A} \cdot k_{\mathrm{eq}}^{A}$ in Eq. (16), the equilibrium constant for the attachment of the ion to the tip of the protrusion at $z_{i}$ in Eq. (12), does include a factor for the formation of the protrusion, $P\left(h_{i}\right) \Delta h_{i} . k_{\text {diff }}$ is the diffusion rate constant for passage from $z_{i}$ to $z_{f}$ and is given by the right-hand side of Eq. (5). $K_{\text {eq }}^{B}$ in Eq. (16), the equilibrium constant for the formation of the ion attached to the protrusion of $A$ extending into bulk $B$, contains the probability of having an $h_{f}$ in $\left(h_{f}, h_{f}+\Delta h_{f}\right)$ and also includes a factor $\exp \left\{-\left[\bar{\mu}\left(z_{f}\right)\right.\right.$ $\left.\left.-\bar{\mu}\left(z_{i}\right)\right] / k_{B} T\right\} ; k_{\text {diss }}^{B}$ is the rate constant for dissociation of the ion from the tip. As the ion diffuses into solvent $B$, the last vestiges of $A$ form a protrusion on whose tip the ion resides.

If the attachment rate at $h_{i}$ were the slow step, $k_{\text {rate }}$ in Eq. (16) would equal the $k_{\text {assn }}^{A}$ and would become independent of the applied potential, giving rise to a Tafel slope of zero. Again, if the detachment rate at $h_{f}$ were the slow step, the $k_{\text {rate }}$ would equal $K_{\mathrm{eq}}^{B} k_{\text {diss }}^{B}$, and the $k_{\text {rate }}$ would have a Tafel slope of unity because of the behavior of $K_{\text {eq }}^{B}$. Approximate (order-of-magnitude) statistical mechanical estimates of the terms in Eq. (16) are made as follows:

For comparison, the transition state value of $k^{A}$ for an idealized interface which is flat rather than having protrusions is considered first, followed by some remarks on a hydrodynamic effect, reflected in a decreasing diffusion constant in close approach to the interface. For the flat surface case $k^{A}$ is, in terms of transition state theory, 


$$
k^{A}=\frac{k_{B} T}{h} \frac{Q^{\dagger}}{Q_{t}} e^{-w_{A}^{P \dagger} / k_{B} T} \quad \text { (plane), }
$$

where $w_{A}^{P \dagger}$ is defined in Table I, $Q^{\dagger}$ is the partition function of the ion per unit $z$, for an ion confined to a unit area of the interface, and $Q_{t}$ is that of the freely moving ion in solvent $A$ just outside the interfacial region, per unit volume,

$$
Q^{\dagger}=\frac{2 \pi m k_{B} T}{h^{2}} g, Q_{t}=\frac{\left(2 \pi m k_{B} T\right)^{3 / 2}}{h^{3}},
$$

where we have introduced the factor $g .{ }^{91}$ The use of a "per unit $z$ " for $Q^{\dagger}$ arises because of the definition of the $c_{i}$ in Eq. (3) as a number per unit $z$ per unit area. The mass of the ion is $m$.

Equations (18)-(19) yield for this planar interface,

$$
k^{A}=Z g e^{-w_{A}^{P \dagger} / k_{B} T} \quad \text { (plane), }
$$

where $Z$ is given by Eq. (21) and, if the estimate that at least one or two $A$ solvent molecules have to be removed to the bulk $A$ then $w_{A}^{P \dagger}$ might be of the order of the value in Eq. (21),

$$
Z=\left(\frac{k_{B} T}{2 \pi m}\right)^{1 / 2}, e^{-w_{A}^{P \dagger} / k_{B} T} \lesssim \frac{1}{10 g} \text { to } \frac{1}{100 g^{2}} .
$$

That is, $k^{A}$ contains, as expected for this simple model, the usual collision frequency per unit area of the interface, $Z, g$ and a barrier $w_{A}^{P \dagger}$ for removing some solvent $A$ molecules before attachment to the interface.

The equilibrium constant $K_{\text {eq }}^{A}$ for this planar case is also immediately obtained. For the equilibrium $c\left(z_{i}\right) \rightleftarrows c_{i}\left(z_{i}\right)$ between an unattached ion near $z_{i}$ and the attached ion at $z_{i}$ the $K_{\text {eq }}^{A}$ in Eq. (16) is the ratio of the equilibrium $c_{i}\left(z_{i}\right)$ to the equilibrium $c\left(z_{i}\right)$. Since $c_{i}\left(z_{i}\right)$ is a number per unit area per unit area per unit $z$, and so has units of $\mathrm{cm}^{-3}$, and since $c\left(z_{i}\right)$, the concentration of unattached ions at $z_{i}$, also has units of $\mathrm{cm}^{-3}, K_{\mathrm{eq}}^{A}$ is dimensionless. In terms of partition functions, the statistical mechanical expression for $K_{\text {eq }}^{A}$ contains in its denominator the product of the three translational partition functions associated with $c\left(z_{i}\right)$, namely, the $Q_{t}$ in Eq. (19). The numerator contains the product of two translational partition functions for the $(x, y)$ motion per unit area parallel to the surface, $2 \pi m k_{B} T / h^{2}$, the factor $g$, and a per unit length partition function for the translation normal to the interface, $\left(2 \pi m k_{B} T\right)^{1 / 2} / h$. Since only one-half of the $z$-momenta is involved in the definition of $K_{\mathrm{eq}}^{A}$, namely, those having positive $d z / d t$, this z-partition function should be divided by 2 . Thereby, using the ratio of these partition functions the $K_{\text {eq }}^{A}$ for this planar interface is

$$
K_{\mathrm{eq}}^{A}=\frac{1}{2} g e^{-\left(\bar{\mu}^{P}-\bar{\mu}\left(z_{i}\right)\right) / k_{B} T} \quad \text { (plane), }
$$

where $\bar{\mu}^{P}$ denotes the $\bar{\mu}$ of the ion when it touches the planar interface. The $\bar{\mu}^{P}$ thus includes the free energy of displacement of several $A$ molecules so that the ion can touch the planar surface, just as the $w_{A}^{P \dagger}$ in Eq. (18) did.

We consider next the values of $k^{A}$ and $K_{\text {eq }}^{A}$ when there are protrusions generated by capillary waves. We use a transition state model. In the transition state the $(x, y)$ motion of the ion transverse to the tip is taken to be localized. We denote the partition function of these two now-vibration-like coordinates by its classical value $\left(k_{B} T / h v\right)^{2}$, where $v$ is their vibrational frequency. The $z$-ionic motion along the axis of the tip is the reaction coordinate and so does not contribute to $Q^{\dagger}$, but the structural factor $g$ is again present. However, the $Q^{\dagger}$ now includes the $P\left(h_{i}\right) \Delta h_{i}$ and the surface density $\sigma$ of the protrusions. Thereby, we have

$$
\frac{k_{B} T}{h} \frac{Q^{\dagger}}{Q}=\frac{k_{B} T}{h}\left(\frac{k_{B} T}{h v}\right)^{2} \frac{g \sigma P\left(h_{i}\right) \Delta h_{i}}{\left(2 \pi m k_{B} T\right)^{3 / 2} / h^{3}}
$$

and it follows that

$$
k_{\mathrm{assn}}^{A}=Z g \sigma P\left(h_{i}\right) \Delta h_{i}\left(\frac{k_{B} T}{h v}\right)^{2} \frac{e^{-w_{A}^{\dagger} / k_{B} T}}{2 \pi k_{B} T / h^{2}}
$$

where $w_{A}^{\dagger}$ is defined in Table I. Upon writing $v=(1 / 2 \pi)$ $\times(k / m)^{1 / 2}$, where $k$ is the force constant for the attached ion's motion transverse to the tip, we note that $\left\langle k r^{2}\right\rangle / 2$ $=k_{B} T$, where $\left\langle r^{2}\right\rangle$ is the thermally averaged mean square displacement of the two-dimensional $(x, y)$ oscillator. We have, thereby,

$$
k_{\mathrm{assn}}^{A}=Z g\left\langle\pi r^{2}\right\rangle \sigma P\left(h_{i}\right) \Delta h_{i} e^{-w_{A}^{\dagger} / k_{B} T} .
$$

The pre-exponential factor in Eq. (25) is smaller than that for the planar interface in Eq. (20), but $w_{A}^{\dagger}$ is expected to be much smaller than the $w_{A}^{P^{\dagger}}$, as discussed earlier. When $P\left(h_{i}\right)$ refers to a protrusion unperturbed by the ion, the effect of any attractive interaction (e.g., ion-solvent polarization) on the protrusion can be incorporated into the term arising from $k^{A}$, namely, $\exp \left(-w_{A}^{\dagger} / k_{B} T\right)$. Information on the relative importance of the various contributions in Eq. (25) can be obtained from simulations appropriate to the exchange rate constant $k_{0}$, when they become available.

The equilibrium constant $K_{\text {eq }}^{A}$ for this surface having protrusions of $B$ into $A$ is given (per unit length along $z$, as before) by

$$
K_{\mathrm{eq}}^{A}=\left(\frac{k_{B} T}{h v}\right)^{2} \frac{\left(2 \pi m k_{B} T\right)^{1 / 2} / h}{2\left(2 \pi m K_{B} T\right)^{3 / 2} / h^{3}} g \sigma P\left(h_{i}\right) \Delta h_{i},
$$

where we have again used the translational partition function of the ion per unit length along the $z$-direction to the tip, divided by 2 so as to include only the ions with positive z-velocity. Thereby, using arguments similar to those given above which led from Eqs. (24)-(25), we have

$$
K_{\mathrm{eq}}^{A}=\frac{1}{2} g\left\langle\pi r^{2}\right\rangle \sigma P\left(h_{i}\right) \Delta h_{i}
$$

which is again dimensionless. Equation (27) reduces to the pre-exponential factor Eq. (22) upon setting $\left\langle\pi r^{2}\right\rangle \sigma=1$, and $P\left(h_{i}\right) \Delta h_{i}=1$.

We consider next the $K_{\text {eq }}^{B} k_{\text {diss }}^{B}$ appearing in Eq. (16). For $K_{\text {eq }}^{B}$ the equilibrium constant for forming the ion at the end of the protrusion $h_{f}$ of solvent $A$ extending into $B$, arguments similar to those used above yield

$$
K_{\mathrm{eq}}^{B}=\frac{1}{2} g\left\langle\pi r^{2}\right\rangle \sigma P\left(h_{f}\right) \Delta h_{f} e^{-\left[\bar{\mu}\left(z_{f}\right)-\bar{\mu}\left(z_{i}\right)\right] / k_{B} T},
$$

where the $\left\langle\pi r^{2}\right\rangle$ and the $\sigma$ now refer to the protrusions of $A$ pointing into bulk $B$. 
For the pre-exponential part of $K_{\text {eq }}^{B} k_{\text {diss }}^{B}$ we have, using arguments similar to those given earlier for $k^{A}$ in Eqs. (23)(24),

$$
\frac{k_{B} T}{h} \frac{Q^{\dagger}}{Q}=\frac{k_{B} T}{h}\left(\frac{k_{B} T}{h v}\right)^{2} \frac{g \sigma P\left(h_{f}\right) \Delta h_{f}}{\left(2 \pi m k_{B} T\right)^{3 / 2} / h^{3}} .
$$

As in the case of (23) which led to (25), Eq. (29) yields

$$
K_{\mathrm{eq}}^{B} k_{\mathrm{diss}}^{B}=Z g\left\langle\pi r^{2}\right\rangle \sigma P\left(h_{f}\right) \Delta h_{f} e^{-\left[\bar{\mu}\left(z_{f}\right)-\bar{\mu}\left(z_{i}\right)+w_{B}^{\dagger}\right] / k_{B} T},
$$

where $\sigma$ and $\left\langle\pi r^{2}\right\rangle$ now refer to the protrusion of $A$ into $B$ and $w_{B}^{\dagger}$ is defined in Table I. The right-hand side again has units of $Z$, e.g., $\mathrm{cm} \mathrm{s}^{-1}$.

We note that when $K_{\text {eq }}^{A} k_{\text {diff }}$ in Eq. (16) is the rate controlling term, we have

$$
k_{\text {rate }}=K_{\text {eq }}^{A} k_{\text {diff }}=\frac{1}{2} g\left\langle\pi r^{2}\right\rangle \sigma P\left(h_{i}\right) \Delta h_{i} k_{\text {diff }},
$$

where $k_{\text {diff }}$ has some form such as Eq. (7).

Several approximate models can be considered for $\bar{\mu}(z)$ in Eq. (5) for $k_{\text {diff }}$ such as the linear profile one represented by Eqs. (6)-(8). In those equations the $z_{f}-z_{i}$ may be of the order of $10 \AA$, the sum of the mean lengths of the protrusions $h_{i}$ and $h_{f}$ and an ionic diameter.

The limits of $k_{\text {diff }}$ in Eqs. (7)-(8) are $k_{\text {diff }}$ $\cong 2 u[\exp (-2 u)] D /\left(z_{f}-z_{i}\right)$ when $u \gg 0$, i.e., when the reaction is very uphill, and $k_{\text {diff }} \cong|2 u|[\exp |-2 u|] D /\left(z_{f}-z_{i}\right)$ when $u$ $\ll 0$, i.e., when the reaction is very downhill. In the latter case the $k_{\text {assn }}^{A}$ in Eq. (16) could eventually become the ratedetermining term for $k_{\text {rate }}$ there. We also note from Eqs. (7)(8) that when the driving force $u$ is small, we have

$$
-k_{B} T \frac{\partial \ln k_{\mathrm{diff}}}{\partial e \Delta \varphi}=0.5 \quad\left(\bar{\mu}\left(z_{f}\right)=\bar{\mu}\left(z_{i}\right)\right),
$$

where $\Delta \varphi=\varphi\left(z_{f}\right)-\varphi\left(z_{i}\right)$. This result also applies to the $k_{\text {rate }}$, given by Eq. (31). A bell-shaped profile for $\bar{\mu}(z)$ instead of the linear profile in Eq. (6) is considered in the Appendix.

\section{Approximate numerical estimates}

We first make some order-of-magnitude numerical estimates of several quantities appearing in the preceding equations. In $k^{A}$ appearing in Eq. (20) for a planar interface $Z g$ is about $\mathrm{g} 10^{4} \mathrm{~cm} \mathrm{~s}^{-1}$, so perhaps about $5 \times 10^{4} \mathrm{~cm} \mathrm{~s}^{-1}$. An estimate for the unperturbed $P\left(h_{i}\right) \Delta h_{i}$ appearing in Eq. (25) is

$$
P\left(h_{i}\right) \Delta h_{i} \cong e^{-h_{i}^{2} / 2\left\langle h^{2}\right\rangle} \Delta h_{i} /\left(2 \pi\left\langle h^{2}\right\rangle\right)^{1 / 2} .
$$

If $\left(\left\langle h^{2}\right\rangle\right)$ were $^{58}$ about $5 \AA^{2}$ and if $h_{i}$ were about $5 \AA$, and $\Delta h_{i}$ about $1 \AA$, then this quantity would be of the order of 0.015 . If the surface density of protrusions $\sigma$ were $^{92}$ approximately $1 / 16 \AA^{-2}$ for a bulk correlation length ${ }^{58}$ of the order of $4 \AA$, and if $\left\langle r^{2}\right\rangle$ were of the order of $1 \AA^{2}$, then $\left\langle\pi r^{2}\right\rangle \sigma$ would be of the order of 0.2. In this case, the factor multiplying the $\exp \left(-w_{A}^{\dagger} / k_{B} T\right)$ in Eq. (25) would be about 0.003 multiplied by $g Z$, i.e., $g 10^{4} \mathrm{~cm} \mathrm{~s}^{-1}$, and so $k_{\text {assn }}^{A}$ would equal about $150 \mathrm{~cm} \mathrm{~s}^{-1}$ if $g \sim 5$, apart from the $w_{A}^{\dagger}$ term. If the ion enhances the formation of the protrusions on the appropriate time scale, as a result of ion-dipole forces, it would enhance
$P\left(h_{i}\right)$ and yield a $k_{\text {assn }}^{A}$ somewhat larger than the above. This $k_{\text {assn }}^{A}$ is far higher than the measured rate constants, and so is not rate-controlling.

We consider next the expression for $K_{\text {eq }}^{A}$ appearing in Eq. (27). Comparison of Eqs. (25) and (27), using the above calculation, shows that the pre-exponential factor in Eq. (27) for $K_{\text {eq }}^{A}$ would be about $0.0015 g$. The $k_{\text {diff }}$ given by Eq. (9) when $\bar{\mu}\left(z_{f}\right)=\bar{\mu}\left(z_{i}\right)$, i.e., for a system close to exchange current conditions, is about $100 \mathrm{~cm} \mathrm{~s}^{-1}$ when $z_{f}-z_{i}$ is about 10 $\AA$ and $D \cong 10^{-5} \mathrm{~cm}^{2} \mathrm{~s}^{-1}$, a typical value for bulk solution. Since there is also a desolvation/solvation occurring, and the molecules of the two immiscible liquids necessarily repel each other in the coordination shells, the effective $D$ may be less than this bulk $D$. When the above value of $k_{\text {diff }}$ of 100 $\mathrm{cm} \mathrm{s}^{-1}$ is multiplied by the above order-of-magnitude estimate for $g\left\langle\pi r^{2}\right\rangle \sigma P\left(h_{i}\right) \Delta h_{i}$, namely, 0.015 , perhaps a value of $1.5 \mathrm{~cm} \mathrm{~s}^{-1}$ is estimated in Eq. (31) for $k_{\text {rate }}$. Since the various estimates above indicate that $k_{\text {assn }}^{A}$ and $K_{\text {eq }}^{B} k_{\text {diss }}^{B}$ are not rate-controlling under such conditions, Eq. (16) leads to Eq. (31). Since the $g \sim 5$ was obtained for a very different system, ${ }^{91}$ a value less than 5 might be applicable.

\section{DISCUSSION OF RESULTS}

As a summary of earlier remarks, we note that the present model uses the idea that protrusions provide a mechanism for initiating a stepwise A-desolvation/Bsolvation of the ion. The most probable $h_{i}$ is determined as a compromise between two factors: the decreased initiation rate when $h_{i}$ is too small, and the decreased $P\left(h_{i}\right)$ when $h_{i}$ is too large. The effect of the attachment or detachment of the ion on the probability of a protrusion can be included in the multiplying attachment and detachment rate constants. An effect of an ion on a protrusion is seen in solvent extraction in the water-dragging by an ion as it moves from an aqueous phase into an organic phase. ${ }^{78}$ A cloudiness and supersaturation with the water results. However, the extent to which such an effect occurs under exchange current conditions, rather than under conditions of large driving force, remains to be determined, apparently. The effect of water dragging is clearly visible in simulations ${ }^{64}$ performed at large driving force. We also noted earlier that the effective $D(z)$ for the $k_{\text {diff }}$ in Eq. (31) may be less than the bulk value, since it is now a diffusion in translation/solvation space.

A third factor which could occur, and when it does will make a bell-shaped profile more appropriate than the linear profile for $\bar{\mu}(z)$ in Eq. (6), arises from the repulsions between unlike solvent molecules. Repulsions lead to a reduced density of nearest neighbor solvent molecules at the interface and so to a reduced solvation of the ion there and hence to a profile for $\bar{\mu}(z)$ which has a maximum between $z_{i}$ and $z_{f}$, instead of being a linear function of $z$. A reduced density at the interface has been seen with light scattering experiments $^{45}$ and in a computer simulation of a model system. ${ }^{66(a)}$ This reduced number density was also reflected in an increase in the transverse diffusion constant of a solvent molecule near the interface. ${ }^{66(\mathrm{~b})}$ When necessary, the effect of a reduced density on $\bar{\mu}(z)$ can be included in Eq. (1) and hence in Eq. (5), by appropriate choice of the func- 
tion $\bar{\mu}(z)$. However, in the computer simulation of $\mathrm{Me}_{4} \mathrm{~N}^{+}$ at a nitrobenzene/water interface there was only a monotonic behavior of $\mu(z)$ vs $z$, and so no maximum in $\mu(z){ }^{64}$

We have commented earlier on the possibility that some $k$ 's deduced from experiments in the literature may be underestimates, because of possible masking by diffusion control or because some resistance other than charge transfer was being measured. We first consider the data from the viewpoint that they are legitimate, and then consider an alternative viewpoint.

One of the (apparent) experimental features, noted earlier, is the remarkably small value of the phenomenological $D$ that is required when the experimental $k_{\text {rate }}$ is equated in the literature to $k_{\text {diff }}$, and a linear profile is used for the $\bar{\mu}(z)$ in Eq. (15). For the $D$ a factor of the order of $1 / 1000$ of the bulk $D$ value was needed to obtain agreement with the "observed" $k_{0}{ }^{8}$ "The result can be explained by effects such as those mentioned above.

The common behavior of having a Tafel coefficient for ion transfer across a liquid/liquid interface of about 0.5 , when the electrochemical driving force is small, is consistent with Eqs. (7) and (31), as well as with various other models. Some estimate has been made of the effect of the linear profile for $\bar{\mu}(z)$, on the plot of $\ln k_{\text {rate }}$ vs the potential across the interface. ${ }^{8}$ In an experiment ${ }^{8}$ the Tafel slope, changed rapidly with the applied potential. It is seen there that when $u$ is increased from 0 to $2, k_{\text {diff }}$ decreases by a factor of $2 \exp (-2) / \sinh 2$, i.e., a factor of 5 , from its value at $u=0$. If the $k_{\text {app }}$ in Eq. (4) is approximated by $k_{\text {rate }}$ there the observed behavior is consistent with Eq. (7) and so with Eq. (31).

The effect of the electrolyte on $k_{0}$ when there is no adsorption of the ion or of the base electrolyte at the interface is of considerable interest. For the moment, we adopt a pragmatic approach based on the "legitimate $k$ " interpretation of the experiments, which is that there is little or no effect of added electrolyte on $k_{0}$, and return to this question below. Thereby, from the point of view of the driving force for the transferring ion across the interface, namely, the potential across the "inner layer" from $h_{i}$ to $h_{f}$, is approximately the total applied potential, and so then $k_{\text {app }} \cong k_{\text {rate }}$ in Eq. (4).

We consider next the experimental observation ${ }^{7}$ that for different ions whose range of $\mu_{B}-\mu_{A}$, i.e., of standard ion transfer potentials, was from $-7 k_{B} T$ to $+5 k_{B} T$, the rate constant $k_{0}$ hardly varied. The $k_{0}$ 's for the transfer of these ions, such as $\mathrm{PF}_{6}^{-}, \mathrm{ClO}_{4}^{-}, \mathrm{BF}_{4}^{-}, \mathrm{SCN}^{-}$, and $\mathrm{Cs}^{+}$and tetraalkyl ammonium ions, across a water/nitrobenzene interface, lay between 0.05 and $0.15 \mathrm{~cm} \mathrm{~s}^{-1}$, depending on ion. ${ }^{7}$ All $\alpha$ values (Tafel slope) at $\bar{\mu}_{A}=\bar{\mu}_{B}$ were around 0.5 . The lack of dependence of the value of $k_{0}$ on the sign of $\mu_{B}$ $-\mu_{A}$ is consistent with both Eq. (9) and Eq. (31), since the diffusion constant $D$ does not explicitly depend on the sign of $\mu_{B}-\mu_{A}$, although the solvation/desolvation which the $D$ involves might be "intrinsically" ion-dependent. In the case of methyl and ethyl orange across the 1,2-dichloroethane (DCE)/water (W) interface, ${ }^{9}$ studied by a different spectroscopic method, the $k_{0}=0.03 \mathrm{~cm} \mathrm{~s}^{-1}$ for both solutes and the $\alpha$ was 0.5 at $\bar{\mu}_{A}=\bar{\mu}_{B}$. This work confirmed earlier reported results based on cyclic voltammetry and ac impedance and a spectroscopic approach.
In a different study ${ }^{8}$ the transfer of the Eosin $\mathrm{Y}$ dianion $\left(E Y^{-2}\right)$ across a DCE/W interface, one charge of $E Y^{-2}$ being localized on a carboxylate group and the other on the xanthene ring, $\alpha=0.5$ at $\bar{\mu}_{A}=\bar{\mu}_{B}$ and $k_{0}$ is 9.5 $\times 10^{-3} \mathrm{~cm} \mathrm{~s}^{-1}$, while the $k_{0}$ for the transfer of tetraethyland tetramethyl-ammonium ions across a nitrobenzene (NB)/W interface was a factor of 10 higher. The ratio of the diffusion constants is a factor of 5, so the $\mathrm{EY}^{2-}$ encountered more additional resistance on crossing the interface than did these tetraalkyl-ammonium ions. However, this factor of 2 difference in the $k_{0} / D$ ratio can have varied causes, such as differences in ease of desolvation/resolvation. Perhaps what is remarkable in a "legitimate $k$ ' interpretation, is the apparent relative insensitivity of all of the various results the value of $k_{0}$ to the effect of $\mu_{B}-\mu_{A}$, the electrolyte concentration, and the nature of the ion crossing the interface.

We consider next the $\bar{\mu}\left(z_{i}\right)-\bar{\mu}_{A}$ (Table I), since this quantity relates $k_{\text {rate }}$ to $k_{\text {app }}$. In treatments of capacitance data at a liquid/liquid interface a modified PoissonBoltzmann equation and other approaches have been used for the diffuse layers, so as to deduce from capacitance data information about the capacitance of the inner layer, the immediate region of the interface, e.g., Ref. 39. In this way the potential difference across the inner layer has been estimated to be of the order of $1 / 3$ the total applied potential, the other $2 / 3$ being the total for the two diffuse double layers. The contribution of the inner layer at a liquid/liquid interface, in the presence of added electrolyte, is estimated from the calculations to be substantially larger than that for a metal/ liquid interface. This result has been attributed to the interpretation of protrusions of each liquid into the other liquid. The protrusions reduce the mean separation distance of the ionic charges on the two sides of the mean local interface, ${ }^{39}$ and since the inner layer capacitance varies inversely with this separation distance, they increase the capacitance.

Such calculations on the diffuse double layers have been used to estimate the effect of the electrolyte on the rate of an ion transfer across the liquid/liquid interface, e.g., by using the data to estimate $\bar{\mu}\left(z_{i}\right)-\bar{\mu}_{A}$ but have encountered the following problem. In Frumkin theory the electrostatic work to bring the ion to the edge of the inner layer $\bar{\mu}\left(z_{i}\right)-\bar{\mu}_{A}$ or at least its electrostatic part is assumed to equal $e \varphi_{2}$, the potential calculated in the absence of that ion. In treating experiments, the use of Frumkin theory has led to too high a computed effect of the electrolyte on the rate constant. In practice the experimental rate constants $k_{0}$ have been insensitive or only weakly sensitive to the electrolyte concentration, in contrast with the Frumkin expression. Some compensation $^{7}$ of the Frumkin effect occurs, due to an opposite effect on the actual driving force on the ion transfer (the $\Delta G^{\circ}$ across the interface), the compensation depending in part on the Tafel coefficient.

Both Gouy-Chapman and modified Poisson-Boltzmann theory have been used to treat capacitance data. The main difference in results between GC and MPB is that the value calculated for $\varphi_{2}$ is smaller when MPB is used, as expected from the nature of a modification in MPB from GC, namely, the presence of a finite size of the ions in the base electrolyte. With this finite size, the large local ion concentrations in GC 
are reduced, leading to a reduced charge density in solution and so to a reduced calculated value of $\varphi_{2}$. The discrepancy between the absence of an effect on $k_{0}$ of added electrolyte and that anticipated on $\varphi_{2}$ and Frumkin theory calculations is reported as only $20 \%$, however. ${ }^{8}$ Nevertheless, in addition to existing computer simulations of double layers for comparing with capacitance data it would be useful to have simulations or analytic theory which estimate better the work term $\bar{\mu}\left(z_{i}\right)-\bar{\mu}_{A}$.

We consider next the opposing view, namely that the $k$ 's obtained in the many studies using techniques such as ac impedance, chronofluorometry or chronoabsorptometry with conventional size electrodes to determine ion transfer rates are in error, in that the measurements are in a region where the process is diffusion controlled. While parameters calculated in Sec. II A do not appear to support this view, it remains a possibility, as does the possibility that some resistance other than charge transfer is also being measured. Such a view could explain the absence of an effect of added electrolyte on $k_{0}$ and the insensitivity (apart from $D$ ) of $k_{0}$ to the nature of the ion and to its free energy of transfer. It will be interesting to see what future measurements at times shorter than the present $0.01 \mathrm{~s}$ or so may reveal.

Computer simulations in the presence of added electrolyte and applied fields, for treating the transfer of an ion across a liquid-liquid interface, will be very interesting for examination of $k_{0}$ and of $k$ in general. As noted earlier, while the simulations made thus far have been very revealing, the observation of fingers or cones, for example, they have not yet been made for the conditions which correspond to actual experiments, with the fields and electrolyte used to balance the difference in Gibbs energy of ion transfer. Such simulations can also provide details on the attachment of the ion to protrusions and whether, in fact, a protrusion is essential in this case. Suggestions were made in an earlier section for remedying this shortcoming, namely, to use in the simulations ions in real liquids which have zero or near zero Gibbs energy of transfer, such as $\mathrm{PF}_{6}^{-}$or $\mathrm{Me}_{4} \mathrm{P}^{+}$in water/ nitrobenzene or $\mathrm{Et}_{4} \mathrm{~N}^{+}$in water/1,2-dichloroethane, for calculation of $k_{0}$, thus avoiding the need for using a more complicated system containing applied fields and supporting electrolyte.

We referred earlier to the interesting simulation ${ }^{66(a)}$ for an admittedly artificial Lennard-Jones system, but one with the considerable virtue of having zero Gibbs energy of ion transfer. The diffusion constant needed to agree with the observed rate constant for ion transfer using Kramers' theory was the bulk value. The barrier to crossing in the system was shown to be due to a reduced solvation. Apparently, either protrusions or slow solvation/resolvation were not a factor in this case, or the computer data were not examined from this viewpoint. It would be useful to make a more local analysis, such as that used in Refs. 58 or 65, which reveal the role, if any, of protrusions. In terms of actual experiments, an activation barrier theory did not appear to give as good agreement with the current vs applied potential data ${ }^{8}$ as did that using Eq. (7) alone. However, more extensive experimental data are clearly needed to explore this question, which remains open. As noted earlier, in another computer simulation ${ }^{64}$ on a realistic rather than model system, $\mathrm{Me}_{4} \mathrm{~N}^{+}$ across a water/nitrobenzene interface, no barrier was found for the locally equilibrated system.

In this latter study, a field of $0.5 \mathrm{~V}$ per $10 \AA$ was added to reduce the trajectory time to $\sim 200$ ps. ${ }^{64}$ Because of this short time, short in comparison with the time inferred from a $k_{0} \sim 0.1 \mathrm{~cm} \mathrm{~s}^{-1}$ and a distance of $10 \AA$, i.e., $1 \mu \mathrm{s}$, the ion did not have time to shed its solvent layers on crossing from bulk A to bulk B. The time required for the shedding was $\sim 100$ ps. ${ }^{64}$ These results are instructive since they reflect a slowness in desolvation. To obtain simulation conditions closer to those involved in $k_{0}$ measurements, it will probably be necessary to study the behavior of an ensemble in which the ion is initially sited at the interface.

In Eqs. (25) and (31) it is presumed that a transition state model can be used to calculate $k_{\text {assn }}^{A}$. If the approach to the ion-protrusion contact were diffusion-limited because of a hydrodynamic-based reduction of the diffusion coefficient, as discussed earlier, then this approach could become rate controlling. However, we have already commented on compensating effects, which make a transition state type model for for $k_{\text {assn }}^{A}$ a reasonable one.

In conclusion, one can identify several possible sources of the smallness of the phenomenological diffusion coefficient $D$ in Eq. (5) for crossing the interface: (1) the possible smallness of the factor $P\left(h_{i}\right) \Delta h_{i}\left\langle\pi r^{2}\right\rangle \sigma$, associated with the protrusions as a way of initiating the ion transfer, (2) the possible smallness of the $D(z)$ present in Eq. (5), small because of a simultaneous concerted replacement of A by B as near neighbors of the ion, and (3) a barrier to the transfer due to a decreased number density in the interfacial region, which would influence the $\bar{\mu}(z)$ function in Eq. (5).

To distinguish experimentally between a low solvation/ spatial diffusion constant $D$ in $k_{\text {diff }}$ in Eqs. (31) and (7) and the protrusion based term there represents a challenge. The probability of fluctuations depends on the interfacial tension, $\gamma$, but other properties besides $\gamma$, also vary when the solvents are varied. Results from appropriate computer simulations on such questions will, of course, be helpful in identifying the principal factors under conditions of zero electrochemical Gibbs energy of transfer. The applied potential effect mentioned earlier for experiments can, in principle, distinguish factor (3) from (1) and (2), i.e., a barrier from nonbarrier theory.

Perhaps studies in which a photon ionizes a solute molecule adsorbed at a liquid/liquid interface, followed by realtime (pico- or femtosecond) observations of the ion as it proceeds into the polar liquid, will provide more direct experimental information on the ion transfer process. It should be emphasized that at the moment we do not know, for conditions where $k \cong k_{0}$, which of the two possibilities described above is the more likely — attachment to a flat interface with a larger barrier $w_{A}^{P \dagger}$ to be overcome, or attachment to a protrusion, with its reduced probability $\left\langle\pi r^{2}\right\rangle \sigma P\left(h_{i}\right) \Delta h_{i}$.

\section{ACKNOWLEDGMENTS}

It is a pleasure to acknowledge the support of this research by the Office of Naval Research and the National 
Science Foundation. It is a pleasure, too, to acknowledge the very helpful correspondence with F. Anson, I. Benjamin, H. H. Girault, T. Kakiuchi, V. Marecek, and Z. Samec, and to dedicate this paper to Roger Parsons on the occasion of his retirement from the editorship of the Journal of Electroanalytical Chemistry.

\section{APPENDIX: BELL-SHAPED PROFILE FOR $\bar{\mu}(z)$}

We consider here a bell-shaped profile of $\bar{\mu}(z)$. Elsewhere an approximate functional form for treating a bondbreaking/bond-forming reaction, $\mathrm{X}_{1} \mathrm{Y}+\mathrm{X}_{2} \rightarrow \mathrm{X}_{1}+\mathrm{YX}_{2}$, was given in terms of an intrinsic barrier and a driving force. ${ }^{93} \mathrm{In}$ the present case there is instead a desolvation of the ion from $\mathrm{A}$ and the concurrent solvation by B. Adapted to the present notation for $k_{\text {diff }}$ it would yield, if $\bar{\mu}(z)$ in Eq. (5) were replaced by its maximum value along $z$,

$$
k_{\mathrm{diff}}=\frac{D p}{z_{f}-z_{i}} \exp \left[-\left(\bar{\lambda}+u+\frac{\bar{\lambda}}{\ln 2} \ln \cosh \frac{u \ln 2}{\bar{\lambda}}\right)\right],
$$

where $u$ is given by Eq. (8) and $\bar{\lambda}$ is the free energy barrier when $u=0$. The $p$ in Eq. (A1) is a prefactor arising because of the replacement of the integrand in Eq. (15) by its maximum. When $u$ is zero or small, this $p$ is unity or close to unity, but when instead the integrand varies greatly, a steepest descent approximation is used and provides an expression for $p$ in this case, $p$ now being a factor less than unity. We omit deriving an expression for the latter. We note that now $k_{\text {diff, }}^{0}$, the value of $k_{0}$ at $u=0$, is given by

$$
k_{\mathrm{diff}}^{0}=\frac{D e^{-\bar{\lambda}}}{z_{f}-z_{i}},
$$

and so is less than that given in Eq. (9) by a factor $\exp (-\bar{\lambda})$. When $u$ becomes large and positive, i.e., when the reaction becomes very uphill, Eq. (A2) yields

$$
k_{\text {diff }}=\frac{D p}{z_{f}-z_{i}} e^{-2 u} \quad(u \gg 0)
$$

while when it becomes very downhill it becomes

$$
k_{\text {diff }}=\frac{D p}{z_{f}-z_{i}} \quad(u \ll 0) \text {. }
$$

${ }^{1}$ Examples of reviews are (a) P. Vanysek, Electrochim. Acta 40, 2841 (1995); (b) H. H. Girault, in Modern Aspects of Electrochemistry, edited by J. O'M. Bockris, B. E. Conway, and R. E. White (Plenum, New York, 1993), Vol. 25, p. 1; (c) I. Benjamin, Annu. Rev. Phys. Chem. 48, 407 (1997); (d) J. Koryta, in Ion-Transfer Kinetics, edited by J. R. Sandifer (VCH, New York, 1995), p. 1; (e) H. H. Girault and D. J. Schiffrin, in Electroanalytical Chemistry, edited by A. J. Bard (Dekker, New York, 1989), Vol. 15, p. 1; (f) Z. Samec and T. Kakiuchi, in Advances in Electrochemistry and Electrochemical Science, edited by H. Gerischer and C. W. Tobias (VCH, Weinheim, 1995), p. 297; (g) I. Benjamin, Chem. Rev. 96, 1449 (1996); (h) Z. Samec, in Liquid-Liquid Interfaces: Theory and Methods, edited by A. G. Volkov and D. W. Deamer (Chemical Rubber, Boca Raton, 1996), p. 155; (i) The Interface Structure and Electrochemical Processes at the Boundary Between Two Immiscible Liquids, edited by V. E. Kazarinov (Springer, Berlin, 1987); (j) K. B. Eisenthal, Chem. Rev. 96, 1343 (1996).

${ }^{2}$ T. Wandlowski, V. Marecek, K. Holub, and Z. Samec, J. Phys. Chem. 93, 8204 (1989).
${ }^{3}$ T. Kakiuchi, Y. Teranshi, and K. Niki, Electrochim. Acta 40, 2869 (1995).

${ }^{4}$ Z. Samec, T. Kakiuchi, and M. Senda, Electrochim. Acta 40, 2971 (1995).

${ }^{5}$ T. Kakiuchi and Y. Teranshi, J. Electroanal. Chem. 396, 401 (1995).

${ }^{6}$ A. Lhotsky, K. Holub, P. Neuzil, and V. Marecek, J. Chem. Soc., Faraday Trans. 92, 3851 (1996).

${ }^{7}$ Z. Samec, Electrochim. Acta 44, 85 (1998).

${ }^{8}$ T. Kakiuchi and Y. Takasu, J. Phys. Chem. B 101, 5963 (1997).

${ }^{9}$ Z. Ding, F. Reymond, P. Baumgartner, D. J. Fermin, P.-F. Brevet, P.-A. Carrupt, and H. H. Girault, Electrochim. Acta 44, 3 (1998).

${ }^{10}$ M. Senda, Electrochim. Acta 40, 2993 (1995).

${ }^{11}$ T. Kakiuchi, J. Noguchi, and M. Senda, J. Electroanal. Chem. 336, 137 (1992).

${ }^{12}$ V. Marecek, A. Lhotosky, and S. Racinsky, Electrochim. Acta 40, 2905 (1995); 40, 2909 (1995)

${ }^{13}$ P. D. Beattie, A. Delay, and H. H. Girault, Electrochim. Acta 40, 2961 (1995).

${ }^{14}$ V. Maracek, M. Gratzl, A. Pungor, and J. Janata, J. Electroanal. Chem. 266, 239 (1989); V. Marecek, M. Gratzl, and J. Janata, ibid. 296, 537 (1990).

${ }^{15}$ J. Josserand, J. Morandini, H. J. Lee, R. Ferrigno, and H. H. Girault, J. Electroanal. Chem. 468, 42 (1999).

${ }^{16}$ T. Wandlowski, V. Marecek, Z. Samec, and R. Fuoco, J. Electroanal. Chem. 331, 765 (1992); Z. Samec (private communications), cf. Ref. 1(f).

${ }^{17}$ J. A. Manzanes, R. Lahtinen, B. Quinn, K. Kontturi, and D. J. Schiffrin, Electrochim. Acta 44, 59 (1998).

${ }^{18}$ Z. Samec, V. Marecek, J. Weber, and D. Homolka, J. Electroanal. Chem. 126, 105 (1981), see also Ref. 1(f).

${ }^{19}$ H. H. Girault and D. J. Schiffrin, J. Electroanal. Chem. 244, 15 (1988); G. Geblewicz and D. J. Schiffrin, ibid. 244, 27 (1988).

${ }^{20}$ V. J. Cunnane, D. J. Schiffrin, C. Beltran, G. Geblewicz, and T. J. Solomon, Electroanal. Chem. 247, 203 (1988).

${ }^{21}$ Y. Cheng and D. J. Schiffrin, J. Electroanal. Chem. 319, 153 (1991).

${ }^{22}$ Y. Cheng and D. J. Schiffrin, J. Chem. Soc., Faraday Trans. 90, 2517 (1994).

${ }^{23}$ C. Wei, A. J. Bard, and M. V. Mirkin, J. Phys. Chem. 99, 16033 (1995).

${ }^{24}$ M. Tsionsky, A. J. Bard, and M. V. Mirkin, J. Phys. Chem. 100, 17881 (1996).

${ }^{25}$ M. Tsionsky, A. J. Bard, and M. V. Mirkin, J. Am. Chem. Soc. 119, 10785 (1997).

${ }^{26}$ M.-H. Delville, M. Tsionsky, and A. J. Bard, Langmuir 14, 2774 (1998).

${ }^{27}$ B. Liu and M. V. Mirkin, J. Am. Chem. Soc. 21, 8352 (1999).

${ }^{28}$ Z. Ding, D. J. Fermin, P.-F. Brevet, and H. H. Girault, J. Electroanal. Chem. 458, 139 (1998).

${ }^{29}$ H. D. Duong, P. F. Brevet, and H. H. Girault, J. Photochem. Photobiol., A 117, 27 (1998).

${ }^{30}$ D. J. Fermin, H. D. Duong, Z. Ding, P.-F. Brevet, and H. H. Girault, Phys. Chem. Chem. Phys. 1, 1461 (1999).

${ }^{31}$ C. Shi and F. C. Anson, Anal. Chem. 70, 3114 (1998).

${ }^{32}$ C. Shi and F. C. Anson, J. Phys. Chem. B 49, 9850 (1998).

${ }^{33}$ C. Shi and F. C. Anson, J. Phys. Chem. B 103, 6283 (1999).

${ }^{34}$ H. Katano, K. Maeda, and M. Senda, J. Electroanal. Chem. 396, 391 (1995).

${ }^{35}$ Y. Zu, F. R.-F. Fan, and A. J. Bard, J. Phys. Chem. B 103, 6272 (1999).

${ }^{36}$ D. J. Fermin, A. Ding, H. D. Duong, P.-F. Brevet, and H. H. Girault, J. Phys. Chem. B 102, 10334 (1998).

${ }^{37}$ Z. H. Zhang, I. Tsuyumoto, T. Kitamori, and T. Sawada, J. Phys. Chem. B 102, 10284 (1998).

${ }^{38}$ Z. Samec, A. Trojanek, and J. Langmaier, J. Electroanal. Chem. 444, 1 (1998).

${ }^{39}$ T. Wandlowski, V. Marecek, K. Holub, and Z. Samec, Electrochim. Acta 40, 2887 (1995).

${ }^{40}$ K. Y. Lee, T. Chin, D. S. Ching, and E. Mazur, J. Phys. Chem. 97, 12876 (1993).

${ }^{41}$ U.-S. Jeng, L. Esibov, L. Crow, and A. Steyerl, J. Phys.: Condens. Matter 10, 4955 (1998)

${ }^{42}$ Z. Zhang, I. Tsuyumoto, T. Takahashi, T. Kitamori, and T. Sawada, J. Phys. Chem. B 101, 4163 (1997).

${ }^{43}$ A. Zarbakhsh, J. Bowers, and J. R. P. Webster, Meas. Sci. Technol. 10, 738 (1999).

${ }^{44}$ C. M. Pereira, W. Schmickler, F. Silva, and M. J. Sousa, J. Electroanal. Chem. 436, 9 (1997).

${ }^{45}$ I. Tsuyumoto, N. Noguchi, T. Kitamori, and T. Sawada, J. Phys. Chem. B 102, 2684 (1998). 
${ }^{46}$ D. M. Mitrinovic, Z. I. Zhang, S. M. Williams, Z. Q. Huang, and M. L. Schlossman, J. Chem. Phys. 110, 7421 (1999).

${ }^{47}$ S. Ishizaka, S. Habuchi, H. B. Kim, and N. Kitamura, Anal. Chem. 71, 3382 (1999)

${ }^{48}$ I. Benjamin, J. Phys. Chem. A 102, 9500 (1998).

${ }^{49}$ See also, Z. Samec, Yu. I. Kharkats, and Yu. Ya. Gurevich, J. Electroanal. Chem. 204, 257 (1986). Equation (10) there represents an approximate form of the present Eq. (1).

${ }^{50}$ T. Kakiuchi, J. Electroanal. Chem. 322, 55 (1992).

${ }^{51}$ W. Schmickler, J. Electroanal. Chem. 426, 5 (1997).

${ }^{52}$ H. H. Girault and K. A. Muznetsov, J. Electroanal. Chem. 432, 19 (1997).

${ }^{53}$ O. Pecina and W. Schmickler, J. Electroanal. Chem. 450, 303 (1998). While this work discusses, instead, ion transfers at a solid electrode, we cite it because of its relevance to solvent extrusion, as discussed later in the text.

${ }^{54}$ Z. Samec, J. Electroanal. Chem. 99, 197 (1979).

${ }^{55}$ K. Aoki, Electrochim. Acta 41, 2321 (1996). V. J. Cunnane, D. J. Schiffrin, M. Fleischman, G. Geblewicz, and D. Williams, J. Electroanal. Chem. 243, 455 (1988).

${ }^{56}$ I. Benjamin, J. Chem. Phys. 96, 577 (1992).

${ }^{57}$ T. M. Chang and L. X. Dang, J. Chem. Phys. 104, 6772 (1996).

${ }^{58}$ I. Benjamin, Science 261, 1558 (1993).

${ }^{59}$ I. Benjamin, J. Chem. Phys. 97, 1432 (1992).

${ }^{60}$ L. X. Dang, J. Phys. Chem. B 103, 8195 (1999).

${ }^{61}$ K. J. Schweighofer and I. Benjamin, J. Phys. Chem. 99, 9974 (1995).

${ }^{62}$ P. A. Fernandes, M. N. D. S. Cordeiro, and J. A. N. F. Gomes, J. Phys. Chem. B 103, 8930 (1999); P. A. Fernandes, M. N. D. S. Cordeiro, and J. A. N. F. Gomes, J. Mol. Struct.: THEOCHEM 463, 151 (1999).

${ }^{63}$ K. J. Schweighofer and I. Benjamin, J. Electroanal. Chem. 391, 1 (1995).

${ }^{64}$ K. Schweighofer and I. Benjamin, J. Phys. Chem. A 103, 10274 (1999).

${ }^{65}$ A. Pohorille and M. A. Wilson, J. Mol. Struct.: THEOCHEM 284, 271 (1993).

${ }^{66}$ (a) M. Hayoun, M. Meyer, and P. Turq, J. Phys. Chem. B 98, 6626 (1994); (b) M. Meyer, M. Mareschal, and M. Hayoun, J. Chem. Phys. 89, 1067 (1988).

${ }^{67}$ R. A. Marcus, J. Phys. Chem. 94, 1050 (1990).

${ }^{68}$ R. A. Marcus, J. Phys. Chem. 94, 4152 (1990).

${ }^{69}$ R. A. Marcus, J. Phys. Chem. 95, 2010 (1991)

${ }^{70}$ Y. I. Kharkats and A. G. Volkov, J. Electroanal. Chem. 184, 435 (1985).

${ }^{71}$ B. B. Smith, J. W. Halley, and A. Nozik, J. Chem. Phys. 205, 245 (1996).

${ }^{72}$ W. Schmickler, J. Electroanal. Chem. 428, 123 (1997).

${ }^{73}$ T. Kakiuchi, Electrochim. Acta 40, 2999 (1995).

${ }^{74}$ Y. Marcus and A. S. Kertes, in Ion Exchange and Solvent Extraction of Metal Complexes, edited by A. J. Bard (Wiley-Interscience, New York, 1969).

${ }^{75}$ B. H. Honig, W. L. Hubbell, and R. F. Flewelling, Annu. Rev. Biophys. Biophys. Chem. 15, 163 (1986).

${ }^{76}$ C. M. Starks, C. L. Liotia, and M. Halpern, Phase Transfer Catalysis (Chapman and Hall, New York, 1994).

${ }^{77}$ E.g., P. G. Bruce, A. Lisowaska-Oleksiak, P. Los, and C. A. Vincent, J. Electroanal. Chem. 367, 279 (1994).

${ }^{78} \mathrm{H}$. H. Girault (private communication).
${ }^{79}$ H. Brenner, Chem. Eng. Sci. 16, 242 (1961).

${ }^{80}$ A. D. Maude, Br. J. Appl. Phys. 12, 293 (1961).

${ }^{81}$ R. G. Cox and H. Brenner, Chem. Eng. Sci. 22, 1753 (1967).

${ }^{82}$ G. I. Taylor, cited in W. Hardy and I. Bircumshaw, Proc. R. Soc. A 108, 12 (1925).

${ }^{83}$ W. H. Walton, in Aerodynamic Capture of Particles, edited by E. G. Richardson (Pergamon, New York, 1960), p. 59.

${ }^{84}$ (a) For example, the limiting behavior for small $d$ is given by the asymptotic analysis for a solid interface in Eq. (2.48) of Ref. 80 as $[6 \pi \eta a v]\left(1+(a / d)+\frac{1}{5} \ln (a / d)+k\right)$ as $d / a \rightarrow 0$, where $k \cong 0.97$. (b) This problem is related, by symmetry, to that of two equal spheres approaching each other, since the free surface boundary condition in the original problem is obeyed on the plane bisecting their line of centers. The latter problem is singular and so the former is also, behaving as $1 / d$. The asymptotic expression for the force for the two-sphere problem is given by various authors, e.g., R. E. Hansford, Mathematika 17, 250 (1970); D. J. Jeffrey, ibid. 29, 58 (1982); M. D. A. Cooley and M. E. O'Neill, ibid. 16, 37 (1969).

${ }^{85}$ For example, the diffusion constant to a solid planar electrode decreases by a factor of 10 when the leading edge of an ion of radius a is $0.12 a$ from the plane, and a factor of 3 when this distance is $0.5 a$ (Ref. 80). For a radius of $2 \AA$, this factor of 3 occurs when the leading edge of the ion is $1 \AA$ from the electrode. This separation distance is small and has a relatively minor adverse effect on the electron transfer rate, namely a factor of 3 if the fall-off ET rate with distance varies as $\exp (-\beta d)$, where $\beta$ $\equiv 1 \AA^{-1}$

${ }^{86}$ F. P. Buff, A. A. Lovett, and F. H. Stillinger, Phys. Rev. Lett. 15, 621 (1965).

${ }^{87}$ J. S. Rowlinson and B. Widom, Molecular Theory of Capillarity (Clarendon, Oxford, 1982), p. 115.

${ }^{88}$ For example, R. A. Marcus, Adv. Chem. Phys. 106, 1 (1999).

${ }^{89}$ In Ref. 1(h), p. 156.

${ }^{90} \mathrm{We}$ use here the hard sphere radial distribution function at contact of about 5. R. O. Watts and I. J. McGee, Liquid State Chemical Physics (Wiley, New York, 1976), p. 137.

${ }^{91}$ This caging effect should be distinguished from effects which lead to "stick" vs "slip" boundary conditions in the motion of an ion. In L. Bocquet, J.-P. Hansen, and J. Piasecki, J. Stat. Phys. 76, 527 (1994), there is a confirmation at the molecular level of the Stokes' "stick" boundary conditions at the surface of the solute; this recent computer simulation of a hard sphere solute (radius $a$ ) in a solvent of hard spheres (radius $b$ ) in the range $a / b$ of $1-4.5$ confirmed the stick hydrodynamic boundary condition for that system. However, the difference between diffusion constants for a Stokes' "stick" and "slip" boundary conditions is only a factor of 1.5, reflecting the long range nature of hydrodynamic interactions in the bulk fluid streamline potential, having terms which vary as $1 / r$. In contrast, the cage effect or the hydrodynamic effect close to surfaces, are both very large.

${ }^{92}$ See also Fig. 4 in Ref. 63

${ }^{93}$ R. A. Marcus, J. Phys. Chem. 72, 891 (1968). 